\title{
Tierische Insuline - trotz Rückzügen auch weiterhin in der Schweiz erhältlich
}

\author{
A. Maurer, A. Teuscher
}

1 Egger M, Davey Smith G, Imhoof H, Teuscher A. Risk of severe hypoglycaemia in insulin treated diabetic patients transferred to human insulin: A case control study. Br Med J 1991;303:617-21

2 Teuscher A, Berger WG. Hypoglycaemia unawareness in diabetics transferred from beef/porcine insulin to human insulin. Lancet 1987;2(8555):382-5.

3 Berger WG, Keller U, Honegger B, Jaeggi E. Warning symptoms of hypoglycaemia during treatment with human and porcine insulin in diabetes mellitus. Lancet 1989;1(8646):1041-4.

4 Egger M, Davey Smith G, Teuscher AU, Teuscher A. Influence of human insulin on symptoms and awareness of hypoglycaemia A randomised double blind crossover trial.

Br Med J 1991;303:622-6.

5 Richter B, Neises G. Cochrane review. In: The Cochrane Database of Systematic Reviews, Issue 3 2002. Oxford: Update Software. Updated quarterly. http://www.update-software.com/ cochrane/abstract.htm.

Korrespondenz:

ForumInsulinSchweiz FIS

Prof. Dr. med. Arthur Teuscher

Bremgartenstrasse 119

CH-3012 Bern
In den 80er Jahren begann in Europa grossflächig die «Umstellung» der insulinabhängigen Diabetikerinnen und Diabetiker von tierischen monokomponenten («highly purified») Insulinen auf gentechnische, sogenannte Humaninsuline. Der Verkauf wurde stark forciert. Parallel dazu häuften sich Berichte aus verschiedenen europäischen Ländern über plötzlich auftretende unerklärliche Hypoglykämien, welche bisher in dieser Art und in diesem Ausmass nicht beobachtet wurden. Abgeschwächte bis völlig fehlende Hypoglykämiewahrnehmungen führten unter Human- im Vergleich mit tierischem Insulin 3mal häufiger zu bewusstlosen Hypoglykämien mit Hospitalisationen [1]. Diese von uns als «human insulin hypoglycemia unawareness» bezeichneten unerwünschten Arzneimittelwirkungen [2] wurden in zwei prospektiven doppelblinden Studien analysiert, und es zeigte sich, dass unter tierischem Insulin signifikant früher und klarer erkennbare Hypoglykämiewarnsymptome auftraten als unter Humaninsulin [3, 4].

\section{Cochrane Review 2002: "Human" insulin versus animal insulin in people with diabetes mellitus [5]}

\section{Keine Vorteile für Humaninsulin}

Die international reputierte, auf «evidence-based medicine» aufgebaute Cochrane Institution (Oxford UK) veröffentlichte kürzlich eine aktualisierte Beurteilung von 45 (aus 91) prospektiven Studien, welche ihre Kriterien erfüllten. Darunter finden sich 2 Studien von Basel und Bern mit 76 Typ-1-Diabetiker und -Diabetikerinnen [3, 4], total 2156 Teilnehmer.

\section{Die Cochrane-Resultate (gerafft)}

- «Humaninsulin» wurde für die Routinebehandlung des Diabetes mellitus Anfang der 80er Jahre eingeführt, ohne dass ein adäquater Vergleich mit tierischen Insulinprodukten bezüglich Effizienz angestellt wurde.

- Die Stoffwechselkontrolle gemessen mit HbA1c wies unter Humaninsulin 7,4 versus 7,5 bei tierischem Insulin auf.

- Die beiden Insuline unterschieden sich bezüglich Insulinantikörper nicht.
- Viele wichtige patientenorientierte Resultate wie Lebensqualität und Auswirkungen auf Diabeteskomplikationen sowie Mortalität wurden nie untersucht. Humaninsuline wurden zum Markt zugelassen ohne wissenschaftliche Beweise für Vorteile gegenüber tierischen Insulinen, insbesondere porcinen Insulinen [5].

\section{Schlussfolgerungen der Cochrane Reviewer}

- «Humaninsulin» weist gegenüber tierischem Insulin keinen Vorteil auf.

- Dass beim Transfer von tierischem auf «Humaninsulin» vermehrt Hypoglykämien auftraten, gibt zu Besorgnis Anlass.

- Die Verfügbarkeit von tierischem Insulin vor allem in den Entwicklungsländern - ist gefährdet. Breitangelegte Studien sollten die Versorgungssituation in diesen Ländern untersuchen.

- Die Zufriedenheit und Lebensqualität der Patientinnen und Patienten und die mit Diabetes zusammenhängende Morbidität wurden nie in genügender Qualität in randomisierten klinischen Studien geprüft. Die durchgeführten Studien boten keine Möglichkeit, die Patientenerfahrungen mit den beiden Insulinen qualitativ zu erfassen. [Bemerkung AT: mit Ausnahme der beiden randomisierten, doppel- und dreifachblinden Schweizer Studien, die einen Vorteil mit tierischem Insulin bezüglich Lebensqualität ergaben.]

- Die Reviewer warnen davor, dass sich in $\mathrm{Zu}-$ kunft bei der Einführung neuer Pharmazeutika die Geschichte rund um das Humaninsulin wiederholen könnte, wenn über deren Wirkung ungenügend informiert und deren Vorteil nicht genügend geprüft wird.

Wir stellen fest, dass die Mehrheit der mit Humaninsulin behandelten Diabetikerinnen und Diabetiker mit diesen Produkten zufrieden ist. Eine bedeutende Minderheit von etwa 20 Prozent kann jedoch mit Humaninsulin keine ausreichende Lebensqualität und keine gute Diabeteseinstellung erzielen, namentlich weil sie unter Humaninsulin verminderte oder fehlende Hypoglykämiewarnzeichen aufweist, was mit Ängsten und Unsicherheit, eingeschränkter 
Lebensqualität und bewusstlosen Hypoglykämien verbunden ist. In diesen Fällen genügt eine zumindest versuchsweise Umstellung auf tierische Insuline, um zu erfahren, ob die Insulinspezies für die erwähnte Problematik verantwortlich ist. Wenn die klassischen Hypowarnungen unter Humaninsulin abgeschwächt bis völlig fehlend sind, kann auch nach vielen Jahren Diabetesdauer ein erneuter Versuch mit tierischen Insulinen zum Erfolg führen.

Als sichere und seit Jahrzehnten bestens bekannte Produkte weisen tierische Insuline keine Nachteile gegenüber Humaninsulinen auf. Gerade deshalb empfehlen wir, im Rahmen der Diabetesberatung nicht ausschliesslich die Verwendung von Humaninsulinen oder Analoga zu propagieren. Vielmehr sollte den Patientinnen und Patienten in Wahrnehmung der ärztlichen Aufklärungspflicht die Wahl zwischen Humaninsulinen und tierischen Insulinen geboten werden. Aufgrund unserer Studien und langjähriger Praxiserfahrung empfehlen wir die Umstellung von synthetischem Insulin auf tierisches Insulin, wenn die Einstellung unter Humaninsulin zu instabilem Blutzucker und zu abgeschwächter Hypoglykämiewahrnehmung führt. Tierisches Insulin hat somit nach wie vor auch seine Bedeutung als Insulin «erster Wahl».

Sowohl das Bundesamt für Gesundheit BAG als auch kantonale Gesundheitsbehörden unterstützen unsere Auffassung, wonach tierisches Insulin für diejenigen Patientinnen und Patienten, die es wünschen und/oder darauf angewiesen sind, auch weiterhin in den benötigten Formen erhältlich bleibt.

\section{Erhältliche tierische Insuline}

In der Schweiz sind zum heutigen Zeitpunkt noch die folgenden Insuline erhältlich (über Galenica):

Radio DRS 1, Sendung «Espresso vom 31. Juli 2002: Import in die Schweiz sei eingestellt worden.

** Nach 50 Jahren erfolgreicher Anwendung zieht Novo Nordisk Lente-Insulin per 31.10.2003 vom Markt zurück.

6 Owens DR, Vora JP, Heding LG, Luzio S, Ryder RE, Atiea J, Hayes TM. Human, porcine and bovine ultralente insulin: subcutaneous administration in normal man.

Diabet Med 1986;3(4):326-9.

7 Conti C. Aufklärungspflicht bei Medikamentenabgabe: neues Bundesgerichtsurteil. Schweiz Ärztezeitung 2002;83(23):1185-6.

\section{CP Pharma (Schweiz) AG}

- Insulin Hypurin Porcine Neutral 10 ml;

- Insulin Hypurin Porcine Isophane 10 ml;

- Insulin Hypurin porcine 30/70 Mix 10 ml.

Diese Insuline sind auch in Packungen von $5 \times 1,5 \mathrm{ml}$ für Autopen erhältlich.

\section{Novo Nordisk}

- Actrapid MC suis $10 \mathrm{ml}$;

- Insulatard MC suis $10 \mathrm{ml}$;

- Lente MC suis/bovin $10 \mathrm{ml}^{* *}$.

- Mixtard $30 \mathrm{MC}$ suis $10 \mathrm{ml}$;

- Semilente MC suis $10 \mathrm{ml}$.
Es ist damit zu rechnen, dass die Produzenten aus Furcht vor den Folgen der BSE-Problematik sämtliche bovinen Pharmaprodukte sehr kurzfristig aus dem Markt zurückziehen werden. Für Patientinnen und Patienten, die weiterhin mit tierischem Insulin behandelt werden möchten, wäre deshalb eine frühzeitige Umstellung von Rinder- auf porcine Insuline in Betracht zu ziehen. Jeder Arzt, jede Ärztin kann aber im Einzelfall bei Swissmedic eine Bewilligung zum Bezug von bovinem Insulin einholen.

Wie unsere Erfahrungen zeigen, ist die heute aktive Ärztegeneration offenbar der Auffassung, es seien in der Schweiz keine tierischen Insuline mehr erhältlich und diese hätten auch keine therapeutische Berechtigung mehr. So werden die tierischen Insuline in der Aus-, Weiter- und Fortbildung an den Universitätskliniken und Regionalspitälern nicht mehr erwähnt. Patientinnen und Patienten wenden sich immer häufiger an uns, weil sie in Arztpraxen, Apotheken und Kliniken sowie bei Diabetes-Gesellschaften die Auskunft erhalten, es gäbe in der Schweiz nur noch Humaninsuline.

Es obliegt dem behandelnden Arzt in Wahrnehmung seiner Aufklärungspflicht, auch auf die Möglichkeit zur Behandlung mit tierischen Insulinen als gleichwertige Therapie hinzuweisen, zumal «Humaninsuline» nachweisbar kinetische Unterschiede gegenüber tierischen Insulinen aufweisen [6] und nach 20 Jahren keine gesicherten Vorteile für die Langzeittherapie bestehen. Schliesslich sollten die auf eine lebenslange Therapie mit diesen Produkten angewiesenen Patientinnen und Patienten frei ihre Wahl für das von ihnen als gut und sicher erkannte Produkt treffen können.

\section{Bundesgerichtsentscheid zur ärztlichen Aufklärungspflicht}

Ein kürzlich ergangenes Bundesgerichtsurteil [7] verdient uneingeschränkte Beachtung und gibt Anlass zu folgenden Bemerkungen:

1. Die vollumfängliche Aufklärung trifft auch diejenigen Ärzte, welche Medikamente verschreiben.

2. Bei der Aufklärung ist namentlich auf Gefahren der künftigen Anwendung sowie auf mögliche Unverträglichkeiten und Nebenwirkungen hinzuweisen. Dies ist auch bei künftiger Selbstmedikation der Fall. Die Insulintherapie kann als eine Art Selbstmedikation betrachtet werden. 
Wir danken unseren Gesundheitsbehörden, dass sie das oben erwähnte Problem erkannt haben und bereit sind, die freie Insulinwahl - auch für die natürlichen tierischen Insuline - in der Schweiz zu gewährleisten und damit einer bedeutenden Minderheit von etwa 20 Prozent Dia- betikerinnen und Diabetikern eine gute Lebensqualität zu erhalten.

NB: Die Autoren stehen in keinem finanziellen Interessenkonflikt.

\section{In manus tuas, medice!}

Das BAG geht davon aus, dass mehr als 10\% der Diabetiker bei der Behandlung mit Humaninsulin keine ausreichende Lebensqualität und keine gute Diabeteseinstellung erzielen, und sieht zudem die Wahlfreiheit des Patienten durch die weltweit sinkende Produktion von tierischen Insulinen gefährdet. Das BAG will sich deshalb für die weitere Sicherung der Herstellung von Schweineinsulin einsetzen, damit betroffenen Diabetikern auch weiterhin die Möglichkeit einer Behandlungsalternative offensteht. BAG-Direktor Prof. Thomas Zeltner hat die WHO auf diese Problematik aufmerksam gemacht und hat sich nach möglichen Massnahmen zur weltweiten Erhaltung von tierischem Insulin erkundigt. Eine Antwort steht noch aus.

Allerdings muss jetzt schon darauf hingewiesen werden, dass unsere Behörden nur sehr begrenzte Möglichkeiten haben, um auf die Produktion von Medikamenten Einfluss nehmen zu können. Sie können auch keine Firma dazu verpflichten, ein registriertes Medikament auf dem Markt zu belassen.

Für die strategischen Überlegungen eines pharmazeutischen Unternehmens zählt letztlich nur der Markt. Angebot und Nachfrage bestimmen das Schicksal eines jeden Medikamentes. Ob die Versorgung mit tierischem Insulin auch weiterhin gesichert werden kann, liegt deshalb zu einem wesentlichen Teil in den Händen der Ärztinnen und Ärzte, welche durch die Verschreibung dieser Medikamente die Nachfrage sicherstellen können. Man kann nur hoffen, dass die Verschreibepraxis nicht dem alleinigen Einfluss des Marketings der Pharmaindustrie unterworfen sein wird, sondern vor allem auf Wissen und Erfahrung beruht.

Dr. med. Markus Trutmann 